\title{
Synchronization Seeking in Multi-agent Dynamic Systems with Parametric Uncertainties
}

\author{
Dongkun Han, Graziano Chesi and Yeung Sam Hung
}

\begin{abstract}
This paper addresses robust consensus problems among multiple agents with uncertain parameters constrained in a given set. Specifically, the network coefficients are supposed polynomial functions of an uncertain vector constrained in a set described by polynomial inequalities. First, the paper provides a necessary and sufficient condition for robust firstorder consensus based on the eigenvalues of the uncertain Laplacian matrix. Based on this condition, a sufficient condition for robust first-order consensus is derived by solving a linear matrix inequality (LMI) problem built by exploiting sumof-squares (SOS) polynomials. Then, the paper provides a necessary and sufficient condition for robust second-order consensus through the uncertain expanded Laplacian matrix and Lyapunov stability theory. Based on this condition, a sufficient condition for robust second-order consensus is derived by solving an LMI problem built by exploiting SOS matrix polynomials. Some numerical examples illustrate the proposed results.
\end{abstract}

\section{INTRODUCTION}

Multi-agent dynamical systems are widely employed to model collective behaviors in various areas, such as fauna movements, insects clustering, sensor communications and robust rendezvous [1]-[4]. Reaching consensus among autonomous agents has attracted numerous attentions and a considerable number of studies have been made to achieve coordinative agreements for dynamic networks [5]-[7]. Traditional research topics focus on the efficiency of algorithms to obtain consensus according to the deterministic dynamical system. On account of the motion of individual vehicle, communication link failure and unexpected noise interfere, an increasing number of studies have focused on transforming network topology. Also, by adopting algebraic graph theory and constructing Erdos-Renyi random graph models, stochastic dynamical systems can be properly simulated, thus providing a foundation for further research on synchronization protocols with ergodic properties. See e.g. [8]-[13] and references therein.

In the basic studies, the dynamical multi-agent networks used to address consensus problems are assumed to be exactly known. Such studies have been generalized by addressing consensus problems in the case of uncertain dynamical multiagent networks. Switching topologies, as a main approach to model altering structures, exploit Laplacian graphs simulating the binomial status of communication links as "on" or "off" in the graph evolving processes [14]-[17]. Other assumptions, such as time delay on information exchange, are investigated to model realistic autonomous multi-vehicle

All authors are with the Department of Electrical and Electronic Engineering, University of Hong Kong, Pokfulam Road, Hong Kong (email:dkhan@eee.hku.hk) systems [18]-[20]. Moreover, nonlinear multi-agent systems with proximity graphs and delayed communications are also considered to model time-varying topological structure [21].

This paper addresses robust consensus problems among multiple agents with uncertain parameters constrained in a given set. Specifically, the network coefficients are supposed polynomial functions of an uncertain vector constrained in a set described by polynomial inequalities. First, the paper provides a necessary and sufficient condition for robust firstorder consensus based on the eigenvalues of the uncertain Laplacian matrix. Based on this condition, a sufficient condition for robust first-order consensus is derived by solving a linear matrix inequality (LMI) problem built by exploiting sum-of-squares (SOS) polynomials (see e.g. [22] about LMIs and [23] and references therein about SOS polynomials). Then, the paper provides a necessary and sufficient condition for robust second-order consensus through the uncertain expanded Laplacian matrix and Lyapunov stability theory. Based on this condition, a sufficient condition for robust second-order consensus is derived by solving an LMI problem built by exploiting SOS matrix polynomials. Some numerical examples illustrate the proposed results.

The paper is organized as follows. Section II provides the problem formulation and some preliminaries about graph theory and SOS polynomials. In Section III, the proposed conditions for robust first-order and second-order consensus are provided. In Section IV, some numerical examples are reported. Lastly, Section V concludes the paper with some final remarks.

\section{Preliminaries}

\section{A. Problem Formulation}

The notation used throughout the paper is as follows:

- $\mathbb{N}, \mathbb{R}$ : natural and real number sets;

- $A^{\prime}$ : transpose of $A$;

- $A>0(A \geq 0)$ : symmetric positive definite (semidefinite) matrix $A$;

- $0_{n}$ : origin of $\mathbb{R}^{n}$;

- $1_{n}=(1, \ldots, 1)^{\prime} \mathbb{R}^{n}$

- $I_{n}: n \times n$ identity matrix;

- $\operatorname{img}(A)$ : image of matrix $A$;

- $\operatorname{ker}(A)$ : null space of matrix $A$.

A group of nodes of multi-agent dynamical system of order $n$ is defined by a finite, nonempty set $\mathscr{A}=\left\{A_{1}, \ldots, A_{n}\right\}$, and the weighted directed graph of $\mathscr{A}$ is described by $\mathscr{G}=$ $(\mathscr{A}, \mathscr{E}, G)$, where $\mathscr{E}$ is the set of directed edges belonging to $\mathscr{A} \times \mathscr{A}$ and $G$ is the weighted adjacency matrix displayed by 
$G=\left(G_{i j}\right)_{n \times n}$. In graph $\mathscr{G}$, a directed edge from $A_{j}$ to $A_{i}$ is defined by $G_{i j}$ and it represents an information transmitting channel from the $j$-th node to the $i$-th node, where vehicle $A_{j}$ and vehicle $A_{i}$ are called parent node and child node, respectively. There is a directed edge in $\mathscr{G}$ if and only if $G_{i j} \neq 0$. The pseudodigraph is considered in this paper to model the communicative topology of multi-agent system with all the edges being positively weighted.

In graph $\mathscr{G}$, a directed path from $A_{i}$ to $A_{j}$ is denoted by a sequence of edges $\left(A_{i}, A_{i 1}\right),\left(A_{i 1}, A_{i 2}\right), \ldots,\left(A_{i l}, A_{j}\right)$ in the directed network with distinct nodes $A_{i k}, k=1, \ldots, l$. A graph $\mathscr{G}$ is a strongly connected graph on the condition that there is a directed path between any pair of distinct notes $A_{i}$ and $A_{j}$ [24]. A root is defined by a node of the property that, for any node $i$ different from $j$, there is a directed path from $i$ to $j$. If graph $\mathscr{G}$ is a directed graph and there is exactly one root and expect the root, every node in $\mathscr{G}$ has exactly one parent, we call $\mathscr{G}$ a directed tree. A spanning tree of a directed graph is a directed tree in which graph edges connect all the nodes of the graph [25]. Hence for a multivertex graph of order $n$, a spanning tree contains $n$ vertices and has $n-1$ edges. If any subset of edges contains or forms a spanning tree, we say that the graph has a spanning tree.

In this paper, we consider uncertain multi-agent dynamical systems. For robust first-order consensus, the continuoustime model for a network with $n$ agents can be expressed by

$$
\dot{x}_{i}(t)=\sum_{j=1, j \neq i}^{n} G_{i j}(\theta)\left(x_{j}(t)-x_{i}(t)\right), \quad i=1, \ldots, n
$$

where $x_{i} \in \mathbb{R}$ is the state of the $i$-th node, $\theta \in \mathbb{R}^{r}$ is an uncertain vector, and $G_{i j}: \mathbb{R}^{r} \rightarrow \mathbb{R}$ is the $(i, j)$-th entry of the uncertain weighted adjacency matrix $G(\theta)=$ $\left(G_{i j}(\theta)\right)_{n \times n}$. The uncertain vector $\theta$ is constrained as

$$
\theta \in \Omega
$$

where

$$
\Omega=\left\{\theta \in \mathbb{R}^{r}: s_{i}(\theta) \geq 0 \forall i=1, \ldots, h\right\}
$$

for some $s_{1}, \ldots, s_{h}: \mathbb{R}^{r} \rightarrow \mathbb{R}$. In the sequel we will assume that the uncertain multi-agent dynamical systems is described by a pseudodigraph, i.e. that $G_{i j}(\theta) \geq 0$ for all $\theta \in \Theta$. Moreover, we will assume that $G(\theta)$ and $s_{1}(\theta), \ldots, s_{h}(\theta)$ are polynomial functions.

The first problem considered in this paper concerns robust first-order consensus, and it is defined as follows.

Problem 1. To establish if, for any initial state, the uncertain multi-agent dynamical system (1) achieves robust first-order consensus, i.e.

$$
\lim _{t \rightarrow \infty} x_{i}(t)-x_{j}(t)=0 \quad \forall \theta \in \Omega .
$$

In order to address this problem, we rewrite the uncertain multi-agent dynamical system (1) as

$$
\dot{x}(t)=-L(\theta) x(t)
$$

where $x=\left(x_{1}, \ldots, x_{n}\right) \in \mathbb{R}^{n}$ is the state vector, and $L(\theta)=$ $\left(L_{i j}(\theta)\right)_{n \times n}$ is the uncertain Laplacian matrix given by

$$
\begin{aligned}
& L_{i j}(\theta)=-G_{i j}(\theta) \forall i \neq j \\
& L_{i i}(\theta)=-\sum_{j=1, j \neq i}^{n} L_{i j}(\theta) .
\end{aligned}
$$

It is worth pointing out that the uncertain Laplacian matrix has the diffusion property that

$$
\sum_{j=1}^{n} L_{i j}(\theta)=0 \quad \forall i=1, \ldots, n .
$$

The second problem considered in this paper concerns robust second-order consensus. Specifically, let us consider the system

$$
\begin{aligned}
\dot{x}_{i}(t)= & \rho_{i}(t) \\
\dot{\rho}_{i}(t)= & \sum_{j=1, j \neq i}^{n} \alpha G_{i j}(\theta)\left(x_{j}(t)-x_{i}(t)\right) \\
& +\sum_{j=1, j \neq i}^{n} \beta G_{i j}(\theta)\left(\rho_{j}(t)-\rho_{i}(t)\right)
\end{aligned}
$$

where $x_{i} \in \mathbb{R}$ is the position state of the $i$-th node, $\rho_{i} \in \mathbb{R}$ is the velocity state of the $i$-th node, and $\alpha, \beta \in \mathbb{R}$ are constants.

Problem 2. To establish if, for any initial state, the uncertain multi-agent dynamical system (8) achieves robust second-order consensus, i.e.

$$
\begin{aligned}
& \lim _{t \rightarrow \infty} x_{i}(t)-x_{j}(t)=0 \\
& \lim _{t \rightarrow \infty} \rho_{i}(t)-\rho_{j}(t)=0 .
\end{aligned}
$$

In order to address this problem, we rewrite the uncertain multi-agent dynamical system (8) as

$$
\begin{aligned}
& \dot{x}_{i}(t)=\rho_{i}(t) \\
& \dot{\rho}_{i}(t)=-\sum_{j=1}^{n} \alpha L_{i j}(\theta) x_{j}(t)+\sum_{j=1}^{n} \beta L_{i j}(\theta) \rho_{j}(t) .
\end{aligned}
$$

By defining the position state vector $x=\left(x_{1}, \ldots, x_{n}\right) \in \mathbb{R}^{n}$, the velocity state vector $\rho=\left(\rho_{1}, \ldots, \rho_{n}\right) \in \mathbb{R}^{n}$ and the global state vector $y=\left(x^{\prime}, \rho^{\prime}\right)^{\prime} \in \mathbb{R}^{2 n}$, this system can be rewritten in compact form as

$$
\dot{y}(t)=\tilde{L}(\theta) y(t)
$$

where $\tilde{L}(\theta)$ is the uncertain extended Laplacian matrix given by

$$
\tilde{L}(\theta)=\left[\begin{array}{cc}
0 & I \\
-\alpha L(\theta) & -\beta L(\theta)
\end{array}\right] .
$$

\section{B. SOS Polynomials}

An useful way of establishing whether a (multivariate) polynomial is nonnegative consists of establishing whether it is a SOS polynomial. In fact, the latter task amounts to solving an LMI problem.

Specifically, let $f(x)$ be a polynomial of degree $2 m$ in $x \in \mathbb{R}^{r}$. Then, $f(x)$ can be written as

$$
f(x)=x^{\{m\}^{\prime}}(F+C(\delta)) x^{\{m\}}
$$


where $x^{\{m\}}$ is a vector containing all monomials of degree less than or equal to $m$ in $x, F$ is a symmetric matrix, and $C(\delta)$ is a linear parametrization of the linear subspace

$$
\mathcal{C}=\left\{C=C^{\prime}: x^{\{m\}^{\prime}} C x^{\{m\}}=0\right\}
$$

The representation (13) is known as Gram matrix method [26] and square matrix representation (SMR) [27]. This representation was introduced in [27] for establishing whether a polynomial is SOS via LMIs. Indeed, $f(x)$ is SOS if there exist polynomials $f_{1}(x), f_{2}(x), \ldots$ such that

$$
f(x)=\sum_{i} f_{i}(x)^{2}
$$

and this condition holds if and only if there exists $\delta$ such that the following LMI feasibility test holds:

$$
F+C(\delta) \geq 0
$$

This techniques can also be used in the case of matrix polynomials. Specifically, let $M(x)$ be a symmetric matrix polynomial of size $s \times s$ of degree $2 m$ in $x \in \mathbb{R}^{r}$ (this means that all the entries of $M(x)$ are polynomials of degree $2 m$ in $x)$. Then, $M(x)$ can be written as

$$
M(x)=\left(x^{\{m\}} \otimes I_{s}\right)^{\prime}(\bar{M}+D(\delta))\left(x^{\{m\}} \otimes I_{s}\right)
$$

where $\bar{M}$ is a symmetric matrix, and $D(\delta)$ is a linear parametrization of the linear subspace

$$
\mathcal{D}=\left\{D=D^{\prime}:\left(x^{\{m\}} \otimes I_{s}\right)^{\prime} D\left(x^{\{m\}} \otimes I_{s}\right)=0\right\} .
$$

The representation (13) was introduced in [28] for establishing whether a matrix polynomial is SOS via LMIs. Indeed, $M(x)$ is SOS if there exist matrix polynomials $M_{1}(x), M_{2}(x), \ldots$ such that

$$
M(x)=\sum_{i} M_{i}(x)^{\prime} M_{i}(x)
$$

and this condition holds if and only if there exists $\delta$ such that the following LMI feasibility test holds:

$$
\bar{M}+D(\delta) \geq 0
$$

It is worth mentioning that SOS polynomials have been exploited in optimization over polynomials since a long time, in particular [29] has been one of the pioneering works on this topic. The reader is also referred to the survey [23] and references therein for details and algorithms about SOS polynomials.

\section{Conditions FOR Robust CONSEnsus}

In this section we derive the proposed conditions for robust first-order and second-order consensus.

\section{A. Robust First-Order Consensus}

For graph $\mathscr{G}=(\mathscr{A}, \mathscr{E}, G)$, it has been found that the consensus of the directed network is determined by the topological structure. The following theorem extends to the case of uncertain multi-agent dynamical systems three existing conditions found for the case of multi-agent dynamical systems without uncertainty [14], and provides a further condition in terms of zeros of a polynomial.

Theorem 1: For a given uncertain Laplacian matrix $L(\theta)$ in (6) and network $\mathscr{G}=(\mathscr{A}, \mathscr{E}, G)$ with uncertain parameters, the following four statements are equivalent.

a) Robust first-order consensus can be achieved.

b) $\forall \theta \in \Omega, L(\theta)$ has exactly one simple eigenvalue 0 and all the other eigenvalues have positive parts.

c) $\forall \theta \in \Omega$, the directed graph $\mathscr{G}$ has a spanning tree.

d) $\forall \theta \in \Omega, q(\theta) \neq 0$, where

$$
q(\theta)=\left.\frac{d}{d \lambda} l(\lambda, \theta)\right|_{\lambda=0}
$$

and

$$
l(\lambda, \theta)=\operatorname{det}(\lambda I-L(\theta)) .
$$

Proof Assume the Laplacian matrix $L(\theta)$ is constructed by (6). Then, the first three statements are equivalent and follow directly from the analogous ones found for the case of multi-agent dynamical systems without uncertainty [14]. From Lemma 3.3 in [14], one has that $\Re\left(\lambda_{i}(L(\theta))\right) \geq 0$, $\forall i=1,2 \ldots, n, \forall \theta \in \Omega$. Moreover, statement d) implies that $L(\theta)$ has exactly one zero eigenvalue, $\forall \theta \in \Omega$. Thus, statements b) and d) are equivalent. Therefore, the theorem holds.

One way of checking condition d) in Theorem 1 consists of using SOS polynomials and amounts to solving an LMI problem. Specifically, define

$$
\begin{gathered}
\qquad c^{*}=\sup _{c, g_{i}(\theta)} c \\
\text { s.t. }\left\{\begin{array}{l}
g_{i}(\theta) \text { is SOS } \\
(-1)^{k} q(\theta)-c-\sum_{i=1}^{h} g_{i}(\theta) s_{i}(\theta) \text { is SOS }
\end{array}\right.
\end{gathered}
$$

where $k \in\{0,1\}$ is defined as

$$
k= \begin{cases}0 & \text { if } q\left(\theta_{0}\right)>0 \\ 1 & \text { otherwise }\end{cases}
$$

and $\theta_{0}$ is any vector $\theta$ in $\Omega$ which can be freely chosen. Then, condition d) in Theorem 1 holds if $c^{*}>0$.

Indeed, it turns out that $c^{*}$ is a lower bound of $q(\theta)$ (if $q\left(\theta_{0}\right)>0$ ) or $-q(\theta)$ (otherwise) for $\theta \in \Omega$. In fact, whenever the constraints in (23) hold, for any $\theta \in \Omega$ it follows that

$$
\begin{aligned}
0 & \leq(-1)^{k} q(\theta)-c-\sum_{i=1}^{h} g_{i}(\theta) s_{i}(\theta) \\
& \leq(-1)^{k} q(\theta)-c
\end{aligned}
$$

i.e. $c$ is a lower bound of $(-1)^{k} q(\theta)$ for $\theta \in \Omega$.

The quantity $c^{*}$ in (23) can be found by solving an LMI problem by using the representation of polynomials reported 
in Section II. Indeed, let $2 m_{i}$ be the degree of $g_{i}(\theta)$ and $2 m_{0}$ be the degree of $(-1)^{k} q(\theta)-c-\sum_{i=1}^{h} g_{i}(\theta) s_{i}(\theta)$. Let us introduce the representations

$$
\begin{aligned}
g_{i}(\theta) & =\theta^{\left\{m_{i}\right\}^{\prime}} G_{i} \theta^{\left\{m_{i}\right\}} \\
g_{i}(\theta) s_{i}(\theta) & =\theta^{\left\{m_{0}\right\}^{\prime}} U_{i}\left(G_{i}\right) \theta^{\left\{m_{0}\right\}} \\
(-1)^{k} q(\theta) & =\theta^{\left\{m_{0}\right\}^{\prime}}(F+C(\delta)) \theta^{\left\{m_{0}\right\}} \\
1 & =\theta^{\left\{m_{0}\right\}^{\prime}} W \theta^{\left\{m_{0}\right\}}
\end{aligned}
$$

where $G_{i}, U_{i}\left(G_{i}\right), F, C(\delta)$ and $W$ are symmetric matrices. Then,

$$
\begin{gathered}
c^{*}=\sup _{c, G_{i}, \delta} c \\
\text { s.t. }\left\{\begin{array}{l}
G_{i} \geq 0 \\
F+C(\delta)-c W-\sum_{i=1}^{h} U_{i}\left(G_{i}\right) \geq 0 .
\end{array}\right.
\end{gathered}
$$

Problem (27) is a convex optimization problem with linear cost function and LMI constraints, known as eigenvalue problem and semidefinite program [22].

\section{B. Robust Second-Order Consensus}

Let us consider the problem of establishing robust secondorder consensus. For this problem, we exploit the uncertain expanded Laplacian matrix $\tilde{L}(\theta)$. First of all, let us introduce the following preliminary result, which extends to the case of uncertain multi-agent dynamical systems the condition given in [20] for the case of multi-agent dynamical systems without uncertainty.

Lemma 1: For all $\theta \in \Omega$, robust second-order consensus for the uncertain multi-agent dynamical system (11) can be obtained if and only if $-\tilde{L}(\theta)$ has only one zero eigenvalue of algebraic multiplicity two and all the other eigenvalues are in the open right half plane.

Starting from this result, we provide a new condition for investigating robust second-order consensus based on matrix inequalities. Specifically, define $w_{1}=1_{n}, w_{2}=0_{n}, w_{3}=$ $0_{n-1}$ and the vectors

$$
u_{1}=\left(\begin{array}{c}
w_{1} \\
w_{2}
\end{array}\right), \quad u_{2}=\left(\begin{array}{c}
w_{3} \\
w_{1}
\end{array}\right) .
$$

Let $V_{1} \in \mathbb{R}^{2 n \times 2 n-1}$ and $V_{2} \in \mathbb{R}^{2 n-1 \times 2 n-2}$ be matrices such that

$$
\operatorname{img}\left(V_{1}\right)=\operatorname{ker}\left(u_{1}^{\prime}\right), \quad \operatorname{img}\left(V_{2}\right)=\operatorname{ker}\left(u_{2}^{\prime}\right) .
$$

Let us define the transformed uncertain expanded Laplacian matrix:

$$
\breve{L}(\theta)=-V_{2}^{\prime} V_{1}^{\prime} \tilde{L}(\theta) V_{1} V_{2} .
$$

Theorem 2: Robust second-order consensus can be achieved if and only if there exists a function $P: \mathbb{R}^{r} \rightarrow$ $\mathbb{R}^{2 n-2 \times 2 n-2}$ such that

$$
\left\{\begin{array}{l}
P(\theta)>0 \\
P(\theta) \breve{L}(\theta)+\breve{L}(\theta)^{\prime} P(\theta)>0
\end{array} \quad \forall \theta \in \Omega .\right.
$$

Proof Let us observe that $u_{1}$ is an eigenvector of $\tilde{L}(\theta)$ corresponding to the eigenvalue zero. Moreover, observe that $V_{1}^{\prime} \tilde{L}(\theta) V_{1}$ has the same eigenvalues of $\tilde{L}(\theta)$ except that the algebraic multiplicity of the eigenvalue zero has been decreased of one. Similarly, it follows that $V_{2}^{\prime} V_{1}^{\prime} \tilde{L}(\theta) V_{1} V_{2}$ has the same eigenvalues of $\tilde{L}(\theta)$ except that the algebraic multiplicity of the eigenvalue zero has been decreased of two. Hence, from Lemma 1, it follows that robust secondorder consensus can be achieved if and only if $-\tilde{L}(\theta)$ has all the eigenvalues in the open right half plane for all $\theta \in \Omega$. From Lyapunov stability theorem for linear systems, this is equivalent to say that there exists $P(\theta)$ such that (31) holds for all $\theta \in \Omega$. Therefore, the theorem holds.

In order to investigate the condition of Theorem 2 , we can exploit SOS matrix polynomials. Specifically, let $P(\theta)$ and $G_{i}(\theta), i=1, \ldots, h$, be symmetric matrix polynomials to be determined, and define

$$
R(\theta)=P(\theta) \breve{L}(\theta)+\breve{L}(\theta)^{\prime} P(\theta)-\sum_{i=1}^{h} G_{i}(\theta) s_{i}(\theta) .
$$

It is easy to verify that (31) holds if there exists $c>0$ such that

$$
\left\{\begin{array}{l}
G_{i}(\theta) \text { is SOS } \\
P(\theta)-I_{2 n-2} \text { is SOS } \\
R(\theta)-c I_{2 n-2} \text { is SOS. }
\end{array}\right.
$$

In fact, whenever the constraints in (33) hold with $c>0$, for any $\theta \in \Omega$ it follows that $G_{i}(\theta) \geq 0, P(\theta)>0$ and

$$
\begin{aligned}
0 & \leq P(\theta) \breve{L}(\theta)+\breve{L}(\theta)^{\prime} P(\theta)-\sum_{i=1}^{h} G_{i}(\theta) s_{i}(\theta)-c I_{2 n-2} \\
& \leq P(\theta) \breve{L}(\theta)+\breve{L}(\theta)^{\prime} P(\theta)-c I_{2 n-2} \\
& \leq P(\theta) \breve{L}(\theta)+\breve{L}(\theta)^{\prime} P(\theta)
\end{aligned}
$$

i.e. (31) holds.

The condition (33) can be formulated via a convex optimization problem with LMI constraints by using the representation of matrix polynomials reported in Section II. Indeed, let $2 m_{i}$ be the degree of $G_{i}(\theta), 2 m$ be the degree of $P(\theta)$, and $2 m_{0}$ be the degree of $R(\theta)-c I$. Let us introduce the representations

$$
\begin{aligned}
G_{i}(\theta) & =\left(\theta^{\left\{m_{i}\right\}} \otimes I_{2 n-2}\right)^{\prime} \bar{G}_{i}\left(\theta^{\left\{m_{i}\right\}} \otimes I_{2 n-2}\right) \\
G_{i}(\theta) s_{i}(\theta) & =\left(\theta^{\left\{m_{0}\right\}} \otimes I_{2 n-2}\right)^{\prime} \bar{U}_{i}\left(\bar{G}_{i}\right)\left(\theta^{\left\{m_{0}\right\}} \otimes I_{2 n-2}\right) \\
P(\theta) & =\left(\theta^{\{m\}} \otimes I_{2 n-2}\right)^{\prime} \bar{P}\left(\theta^{\{m\}} \otimes I_{2 n-2}\right) \\
R(\theta) & =\left(\theta^{\left\{m_{0}\right\}} \otimes I_{2 n-2}\right)^{\prime}(\bar{F}+D(\delta))\left(\theta^{\left\{m_{0}\right\}} \otimes I_{2 n-2}\right)
\end{aligned}
$$

where $\bar{G}_{i}, \bar{U}_{i}\left(\bar{G}_{i}\right), \bar{P}, \bar{F}$ and $D(\delta)$ are symmetric matrices. Then, define

$$
\begin{gathered}
\qquad c^{*}=\sup _{c, \bar{G}_{i}, \bar{P}, \delta} c \\
\text { s.t. }\left\{\begin{array}{l}
\bar{G}_{i} \geq 0 \\
\bar{P} \geq I_{s_{1}} \\
\bar{F}+D(\delta)-c I_{s_{2}}-\sum_{i=1}^{h} \bar{U}_{i}\left(\bar{G}_{i}\right) \geq 0
\end{array}\right.
\end{gathered}
$$

where $s_{1}$ and $s_{2}$ are the sizes of $\bar{P}$ and $\bar{F}$, respectively. Then, it directly follows that (31) holds if $c^{*}>0$. 


\section{NUMERICAL EXAMPLES}

In this section, a couple of simulation examples are provided to illustrate the proposed approach both for firstorder consensus and for second-order consensus.

\section{A. Example 1}

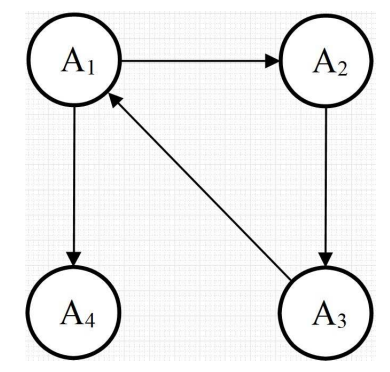

Fig. 1. Multi-agent system considered in Example 1.

In this example we consider the multi-agent system shown in Figure 1. It is assumed that the network is affected by an uncertain parameter, specifically

$$
G(\theta)=\left[\begin{array}{cccc}
1 & 0 & \theta+1 & 0 \\
3 \theta & 1 & 0 & 0 \\
0 & 2-\theta & 1 & 0 \\
3 \theta+2 & 0 & 0 & 1
\end{array}\right]
$$

where $\theta$ is constrained in the set $\Omega$ chosen as

$$
\Omega=[0,1] .
$$

Hence, we have $n=4$ and $r=1$. Moreover, $\Omega$ can be described as in (3) with

$$
s_{1}(\theta)=\theta(1-\theta) .
$$

According to (6), the Laplacian matrix $L(\theta)$ is given by:

$$
L(\theta)=\left[\begin{array}{cccc}
\theta+1 & 0 & -\theta-1 & 0 \\
-3 \theta & 3 \theta & 0 & 0 \\
0 & \theta-2 & 2-\theta & 0 \\
-3 \theta-2 & 0 & 0 & 3 \theta+2
\end{array}\right] .
$$

To establish whether this uncertain network is able to achieve robust first-order consensus, let us use Theorem 1. In particular, the polynomial $q(\theta)$ in (21) is given by:

$$
q(\theta)=3 \theta^{3}+2 \theta^{2}-56 \theta-4 .
$$

According to condition d) in Theorem 1, robust first-order consensus is achieved if and only if $q(\theta) \neq 0$ for all $\theta \in$ $[0,1]$. In this case, it is easy to see that $q(\theta)$ satisfies this property since $q(\theta)$ is an univariate polynomial with all roots outside lying outside $[0,1]$. Nevertheless, let us compute the quantity $c^{*}$ in (27). We have that $k=-1$, and by simply choosing multiplier $g_{1}(\theta)$ of degree 2 we find $c^{*}=4$, which proves that condition d) in Theorem 1 is satisfied.

Next, let us consider the problem of establishing whether this uncertain network is able to achieve robust secondorder consensus. We choose $\alpha=\beta=1$ in the system (8), and we use Theorem 2 by looking for a constant matrix function $P(\theta)$ satisfying (31). By solving (36) we find $c^{*}=$ $+\infty$, i.e. (33) holds with any positive scalar $c$. Therefore, robust second-order consensus is achieved. In this case, the uncertain extended Laplacian matrix is given by

$$
\left[\begin{array}{cccccccc}
0 & 0 & 0 & 0 & 1 & 0 & 0 & 0 \\
0 & 0 & 0 & 0 & 0 & 1 & 0 & 0 \\
0 & 0 & 0 & 0 & 0 & 0 & 1 & 0 \\
0 & 0 & 0 & 0 & 0 & 0 & 0 & 1 \\
l_{1} & 0 & -l_{1} & 0 & l_{1} & 0 & -l_{1} & 0 \\
l_{2} & -l_{2} & 0 & 0 & l_{2} & -l_{2} & 0 & 0 \\
0 & l_{3} & -l_{3} & 0 & 0 & l_{3} & -l_{3} & 0 \\
l_{4} & 0 & 0 & -l_{4} & l_{4} & 0 & 0 & -l_{4}
\end{array}\right]
$$

where $l_{1}=-\theta-1, l_{2}=3 \theta, l_{3}=2-\theta, l_{4}=3 \theta+2$.

\section{B. Example 2}

In this example we consider the uncertain matrix $G(\theta)$ given by

$$
G(\theta)=\left[\begin{array}{cccc}
1 & 2-2 \theta_{2} & 0 & 2 \\
0 & 1 & 1-\theta_{1} & 0 \\
1 & 0 & 1 & 1-\theta_{2} \\
0 & 2-\theta_{1} & 0 & 1
\end{array}\right]
$$

where $\theta \in \mathbb{R}^{2}$ is constrained in the set $\Omega$ chosen as

$$
\Omega=[-1,1]^{2} .
$$

Hence, we have $n=4$ and $r=2$. Moreover, $\Omega$ can be described as in (3) with

$$
s_{i}(\theta)=1-\theta_{i}^{2} \quad \forall i=1,2 .
$$

According to (6), the Laplacian matrix $L(\theta)$ is given by:

$$
L(\theta)=\left[\begin{array}{cccc}
4-2 \theta_{2} & 2 \theta_{2}-2 & 0 & -2 \\
0 & 1-\theta_{1} & \theta_{1}-1 & 0 \\
-1 & 0 & 2-\theta_{2} & \theta_{2}-1 \\
0 & \theta_{1}-2 & 0 & 2-\theta_{1}
\end{array}\right]
$$

To establish whether this uncertain network is able to achieve robust first-order consensus, let us use Theorem 1. In particular, the polynomial $q(\theta)$ in (21) is given by:

$q(\theta)=2 \theta_{1}^{2} \theta_{2}-5 \theta_{1}^{2}+4 \theta_{1} \theta_{2}^{2}-20 \theta_{1} \theta_{2}+29 \theta_{1}-6 \theta_{2}^{2}+26 \theta_{2}-32$.

According to condition d) in Theorem 1, robust first-order consensus is achieved if and only if $q(\theta) \neq 0$ for all $\theta \in$ $[-1,1]^{2}$. Let us compute the quantity $c^{*}$ in (27). We have that $k=-1$, and by simply choosing multiplier $g_{1}(\theta)$ of degree 2 we find $c^{*}=2$, which proves that condition d) in Theorem 1 is satisfied.

Next, let us consider the problem of establishing whether this uncertain network is able to achieve robust second-order consensus. We choose $\alpha=1$ and $\beta=0.6$ in the system (8), and we use Theorem 2 by looking for a constant matrix function $P(\theta)$ satisfying (31). By solving (36) we find $c^{*}=$ -0.252 , which does not prove (33). We repeat the procedure by looking for a matrix function $P(\theta)$ of degree 2 , and we find $c^{*}=+\infty$, i.e. (33) holds with any positive scalar $c$. 
Therefore, robust second-order consensus is achieved. In this case, the uncertain extended Laplacian matrix is given by

$\left[\begin{array}{cccccccc}0 & 0 & 0 & 0 & 1 & 0 & 0 & 0 \\ 0 & 0 & 0 & 0 & 0 & 1 & 0 & 0 \\ 0 & 0 & 0 & 0 & 0 & 0 & 1 & 0 \\ 0 & 0 & 0 & 0 & 0 & 0 & 0 & 1 \\ l_{1} & l_{2} & 0 & 2 & 0.6 l_{1} & 0.6 l_{2} & 0 & 1.2 \\ 0 & l_{3} & -l_{3} & 0 & 0 & 0.6 l_{3} & -0.6 l_{3} & 0 \\ 1 & 0 & 0.5 l_{1} & 0.5 l_{2} & 0.6 & 0 & 0.3 l_{1} & 0.3 l_{2} \\ 0 & l_{4} & 0 & -l_{4} & 0 & 0.6 l_{4} & 0 & -0.6 l_{4}\end{array}\right]$

where $l_{1}=2 \theta_{2}-4, l_{2}=2-2 \theta_{2}, l_{3}=\theta_{1}-1, l_{4}=2-\theta_{1}$.

\section{CONCLUSIONS}

In this paper we have addressed the problems of establishing robust first-order and second-order consensus in uncertain multi-agent dynamical systems. For these problems we have provided necessary and sufficient conditions based on the properties of the uncertain Laplacian matrix and Lyapunov stability theory, and we have derived sufficient conditions that amount to solving LMI problems built by exploiting SOS matrix polynomials.

\section{REFERENCES}

[1] M.H. DeGroot. Reaching a consensus. J. Am. Statistical Assoc., 69(2), 1974.

[2] L. Xiao, S. Boyd, and S. Lall. A scheme for robust distributed sensor fusion based on average consensus. In in proc. 4th Int. Conf. Inf. Process. Sensor Netw., pages 63-70, Los Angeles, CA, April 2005.

[3] S. Boyd, A. Gosh, B. Prabhakar, and D. Shah. Gossip algorithm: Design, analysis and applications. In in Proc. IEEE INFOCOM 2005 pages 1653-1664, Miami, FL, 2005.

[4] J. Cortes, S. Martinez, and F. Bullo. Robust rendevous for mobile autonomous agents via proximity graphs in arbitrary dimensions. IEEE Trans. on Autom. Control, 51(4), 2006.

[5] J.A. Fax and R.M. Murray. Information flow and cooperative control of vehicle formations. IEEE Trans. on Autom. Control, 49(4), 2004

[6] L. Xiao and S. Boyd. Fast linear iterations for distributed averaging. Syst. Control Lett., 53(5), 2004.

[7] C. Wu and L.O. Chua. Synchronization in an array of linearly coupled dynamical system. IEEE Trans. on Circ. and Syst., 42(6), 1995.

[8] L.R. Foulds, editor. Graph Theory Application. Springer-Verlag, 1994.

[9] R.W. Beard and V. Stepanyan. Synchronization of information in distributed multiple vehicle coordinated control. In in Proc. IEEE Conf. Decision and Control, pages 2029-2034, Maui, HI, 2003.

[10] A. Jadbabaie, J. Lin, and A.S. Morse. Coordination of groups of mobile autonomous agents usiing nearest neighbor rules. IEEE Trans. Autom. Control, 48(2), 2003.

[11] C.W. Wu. Synchronization and concergence of linear dynamics in random directed networks. IEEE Trans. Autom. Control, 51(2), 2006.

[12] A. Kashyap, T. Basar, and R. Srikant. Quantized consensus. Automatica, 43(7), 2007.

[13] Y. Tian and C. Liu. Consensus of multi-agent systems with diverse input and communication delays. IEEE Trans. Autom. Control, 53(2), 2008.

[14] W. Ren and R.W. Beard. Consensus seeking in multiagent systems uncer dynamically changing interaction topologies. IEEE Trans. Autom. Control, 50(3), 2005.

[15] A. Tahbaz-Salehi and A. Jadbabaie. A necessay and sufficient consition for consensus over random networks. IEEE Trans. Autom. Control, 53(3), 2008.

[16] Hatano and M. Mesbahi. Agreement over random network. IEEE Trans. Autom. Control, 50(8), 2005.

[17] M. Cao, A.S. Morse, and B.D.O. Anderson. Reaching a consensus in a dynamically changing environment: a graphical approach. SIAM Journal on Control and Optimization, 47(3), 2008. networks of agents with delayed communications. Automatica, 44(2), 2008.

[19] R. Olfati-Saber and R.M. Murray. Consensus problems in networks of agents with swirching topology and time-delays. IEEE Trans. Autom. Control, 49(2), 2004.

[20] W. Yu, G. Chen, and M. Cao. Some necessary and sufficient conditions for second-order consensus in multi-agent dynamical systems. Automatica, 46(4), 2010.

[21] U. Münz, A. Papachristodoulou, and F. Allgöwer. Robust consensus controller design for nonlinear relative degree two multi-agent systems with communication constraints. IEEE Trans. Autom. Control, 56:145151, 2011.

[22] S. Boyd, L. El Ghaoui, E. Feron, and V. Balakrishnan. Linear Matrix Inequalities in System and Control Theory, volume 15 of Studies in Applied Mathematics. SIAM, Philadelphia, PA, June 1994.

[23] G. Chesi. LMI techniques for optimization over polynomials in control: a survey. IEEE Trans. on Automatic Control, 55(11):25002510,2010

[24] C. Godsil and G. Royle, editors. Algebraic Graph Theory. SpringerVerlag, 2001

[25] R.A. Horn and C.R. Johnson, editors. Matrix Analysis. Cambridge Univ. Press, 1985.

[26] M. Choi, T. Lam, and B. Reznick. Sums of squares of real polynomials. In Symposia in Pure Mathematics, pages 103-126, 1995.

[27] G. Chesi, A. Tesi, A. Vicino, and R. Genesio. On convexification of some minimum distance problems. In European Control Conf., Karlsruhe, Germany, 1999.

[28] G. Chesi, A. Garulli, A. Tesi, and A. Vicino. Robust stability for polytopic systems via polynomially parameter-dependent Lyapunov functions. In IEEE Conf. on Decision and Control, pages 4670-4675, Maui, Hawaii, 2003.

[29] N. Z. Shor. Class of global minimum bounds of polynomial functions (in russian). Cybernetics, 23:731-734, 1987. 